\title{
Dynamics and Energy Conversion of Aircraft Landing Gears at Touchdown
}

\author{
W. J. Wang \\ University of Sussex, United Kingdom
}

\begin{abstract}
Dynamics of aircraft landing gears with and without pre-rotation has been analysed. The ground friction work is been formulated which raises the temperature of touch point to the rubber smoke point. The sliding time, distance, power and energy released by the friction at touch point have been presented. Simulation of target speed has been shown for different levels of pre-rotation torque.
\end{abstract}

\section{Introduction}

Hundred thousands of aircraft take off and make their landing everyday worldwide. Up to date, during the touchdown, all aircraft generate smoke, blacken the runway and cause significant tyre wear. No doubt, this behaviour of landing applies damage to environment and increases costs on aircraft operation. Since 1940s, some inventors have made attempt to develop pre-rotation devices and a number of them have been patented [1-2]. However, so far after 80 years past, surprisingly, none of such devices has been put into practical use. Some of the landing gear manufacturers concern about the complicity and additional weight by adding such a device. The landing touchdown smoke is still a standard scene to which most people have got accustomed. With increasing pressure on environment in this 21 st century, action apparently is needed. Aircraft operators provided experience and data for investigators [3]. Rubber tires for vehicles and their friction behaviours are still attractive topics [4-9]. Some have intensively investigated rubber combustion [10]. However, fundamental understanding to the touchdown problem and the measure to reduce the landing smoke are seriously lacking. In recent years, the authors have proposed some fundamental approach to analyse and simulate the dynamic behaviours [11-12] for aircraft touchdown processes. This paper is reflecting the novel but approach to the problem by the author, aiming for eventually getting rid of the landing smoke, increasing the tyre life cycle and protecting the environment.

\section{Landing gear sliding with pre- rotation}

Instantly after the landing gear touches the runway, it is for the ground friction to provide a torque to accelerate the static wheel to match the landing speed within a fraction or a couple of seconds.

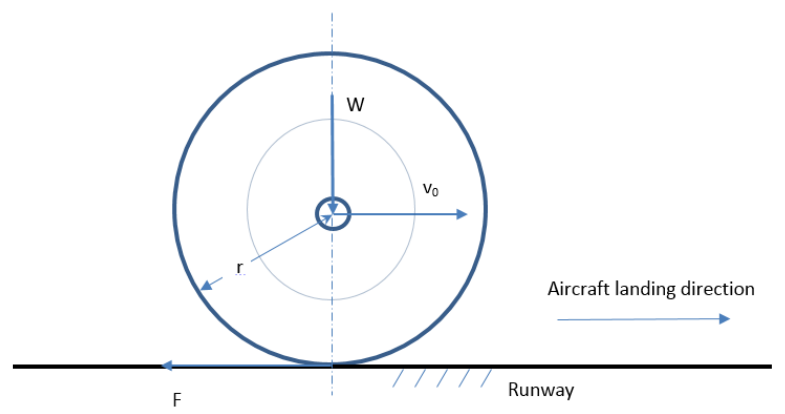

Figure 1. Landing gear wheel at touchdown

Let $v$ be the aircraft travel speed and $\omega$ angular speed of the landing gear wheel. For a landing speed $v=v_{0}$, by a $100 \%$ matching, the tangential speed of wheel at touch point is also $v_{0}=r \omega_{0}$. Assuming the friction provided by the runway ground $F=\mu W$, where $\mu$ is the friction coefficient, depending on type of tyre, contact area and runway condition; $W$ is the vertical force on the wheel shaft due to weight of aircraft as shown in Fig. 1. Normally there are a number, from several to dozens, of wheels for an aircraft. After touchdown, using Newton's second law of motion for rotational circumstances, with a resistance torque $T_{r}$ which includes those in shaft, air viscosity and ground rolling resistances,

$$
F r-T_{r}=I \frac{d \omega}{d t}
$$

where $I$ is the moment of inertia of the wheel, $r$ is the radius of wheel. This radius can be the effective radius, taking into account of tyre deflection. Letting $\varepsilon$ be the 
pre-rotation ratio, i.e., the percentage of landing speed $v_{0}$ being pre-rotated by the device, and letting $t_{s}$ be the sliding time, also assuming the angular acceleration is uniform from static or a slow speed to the matching landing speed $v_{0} / r$,

$$
\frac{d \omega}{d t}=\frac{(1-\varepsilon) v_{0}}{r t_{s}}
$$

It is the friction between the ground and tyre that accelerates the wheel to a landing rotational speed within $t_{s}$ by limited torque $F \cdot r$. The wheel needs a period of time to accelerate to $\omega_{0}$ as the ground can only provide a limited, not unlimited, friction $F$. Therefore, a sliding is inevitable. The sliding time $t_{s}=0$ only if the ground can provide infinitive friction to generate an infinitive acceleration from static to $\omega_{0}$, which is impossible. During this sliding period the wheel is assumed being accelerated uniformly. A side wind turbine is suggested as in the patents to fit on the wheel, on which an air dynamic torque is generated to accelerate the wheel to achieve a tangential speed before the touchdown. After the touchdown, no energy or just smaller energy is required to accelerate the wheel to the matching speed $v_{0}=r \omega_{0}$. From Eqs.(1) and (2)

$$
F r-T_{r}=I \frac{(1-\varepsilon) v_{0}}{r t_{s}}
$$

Therefore, the wheel sliding time is

$$
t_{s}=\frac{(1-\varepsilon) v_{0} I}{r\left(F r-T_{r}\right)}
$$

If the pre-rotation level $\varepsilon=0, t_{s}=\frac{v_{0} I}{r\left(F r-T_{r}\right)}$; if $\varepsilon=1$, $t_{s}=0$. i.e. no sliding exists nor smoke will be generated. The sliding distance, or aircraft travelled distance during sliding is

$$
s_{s}=v_{0} t_{s}=\frac{(1-\varepsilon) v_{0}^{2} I}{r\left(F r-T_{r}\right)}
$$

Since the wheel is in the state of sliding, the tangential motion of wheel at touch point pushed by ground friction $S_{r}$ is less than $S_{s}$.

$$
s_{r}=\frac{(1+\varepsilon) v_{0}}{2} t_{s}=\frac{\left(1-\varepsilon^{2}\right) v_{0}^{2} I}{2 r\left(F r-T_{r}\right)}
$$

The tangential displacement caused by the forces acted on the touch point during the whole sliding period $\left(0 \leq t \leq t_{s}\right)$ is

$$
s=v_{0} t-\frac{(1+\varepsilon) v_{0}}{2} t=\frac{(1-\varepsilon) v_{0}}{2} t
$$

The rate of work done by ground friction, or the friction power, is

$$
\Delta \dot{E}=F \frac{(1-\varepsilon) v_{0}}{2}
$$

It is the power density during the sliding generated by the ground friction on a single tyre. In the case of no prerotation, $\varepsilon=0$, it yields $\dot{E}=\frac{F v_{0}}{2}$. Assuming $F=1.449 \times 10^{4} \mathrm{~N}$ and $v_{0}=80.8 \mathrm{~m} / \mathrm{s}, \Delta \dot{E}=585 \mathrm{~kW}$. This is very powerful figure. In the case of full pre-rotation, $\varepsilon=1$, then $\dot{E}=0$, i.e. the ground friction does not need to do any work on tyres. Using the displacement Eqs. (5) and (6), the ground friction work at the touch point is

$$
\Delta E_{g}=F\left(s_{s}-s_{r}\right)=\frac{(1-\varepsilon)^{2} v_{0}^{2} I F}{2 r\left(F r-T_{r}\right)}
$$

According to the first law of thermodynamics, the energy is conserved. This work will be totally converted to heat energy during the sliding. Using the public domain data from Boeing 747-400 aircraft without prerotation, the moment of inertia of real wheel $I=46.19 \mathrm{~kg}$ $\mathrm{m}^{2}$, the tire radius $r=0.622 \mathrm{~m}$, the landing velocity $v_{0}=80.8 \mathrm{~m} / \mathrm{s}, \Delta E_{\mathrm{g}}$ will be $433 \mathrm{~kJ}$ within the sliding time $t_{s}=0.74$ seconds. Most of it will raise temperature of the rubber near local contact area to its smoke point, generating a large quantity of smoke. The remaining part will be dissipated against the friction $T_{r}$, which is a relatively small number. A tyre is made up of carbon polymer. Primarily, the sliding between tyre and runway surface causes rubber loses. In addition, since the conduction of rubber is poor, this energy will be converted to heat to rise local area temperature at the tyre-ground interface significantly. As a result, the high temperature is to burn the tyre circumference layer to cause the tyre rapid wear and landing smoke. The rubber heat capacity is in average $0.48 \mathrm{KJ} / \mathrm{KgK}$. This energy can cause a high temperature up to $500{ }^{\circ} \mathrm{C}$ on the rubber beyond its ignition point. As a polymer, the flammability depends on oxygen supply and ignition source. Although self-ignition usually does not occur due to strong air flow, it has already entered a temperature range high enough to start chemical oxidization reaction on rubber, causing a large quantity of oxidization smoke. On the other hand, the wheel has gained energy through the sliding from part of the friction work. The kinetic energy gained by the whole wheel is

$$
\Delta E_{w}=\frac{1}{2}\left(1-\varepsilon^{2}\right) I\left(\frac{v_{0}}{r}\right)^{2}
$$

In the case of zero pre-rotation, $E_{w}=\frac{1}{2} I\left(\frac{v_{0}}{r}\right)^{2}$. This energy will be eventually dissipated in the subsequent 
aircraft braking or in air and runway frictions. The total work done by ground friction in sliding is

$$
\Delta E=\frac{(1-\varepsilon)^{2} v_{0}^{2} I F}{2 r\left(F r-T_{r}\right)}+\frac{1}{2}\left(1-\varepsilon^{2}\right) I\left(\frac{v_{0}}{r}\right)^{2}
$$

If without pre-rotation, $\varepsilon=0$, the total energy will be $\Delta E=\frac{v_{0}^{2} I F}{2 r\left(F r-T_{r}\right)}+\frac{1}{2} I\left(\frac{v_{0}}{r}\right)^{2}$. In the case that $T_{r}$ is small and can be ignored, $\Delta E=I\left(\frac{v_{0}}{r}\right)^{2}$. i.e. in this case, the work done by the ground friction is the twice of the kinetic energy gained by the wheel during the sliding.

\section{Pre-rotating touchdown}

After the landing gear is dropped and locked into place, with a pre-rotation device fitted, it is designed to produce a constant torque $T$, when the aircraft is still in air, Newton's law of motion, again, for the wheel is applicable:

$$
T-T_{a}(\omega)=I \frac{d \omega}{d t}=\frac{I}{r} \frac{d v}{d t}
$$

where $T_{a}(\omega)$ is the resistance torque from the shaft bearing due to wheel's aerodynamic drag and weight, and the viscosity from surrounding air. The task of prerotation torque $T$ is to accelerate the wheel from 0 to $\varepsilon v_{0}=\varepsilon r \omega_{0}$. Ideally, when $v_{0}$ is fully reached, the wind turbine torque is just balanced by the friction $T_{a}\left(\omega_{0}\right)$. Assuming the resistance torque applied on the wheel is non-linear $T_{a}(\omega)=\eta \omega^{2}$, from Eq.(12)

$$
T-\eta\left(\frac{v}{r}\right)^{2}=\frac{I}{r} \frac{d v}{d t}
$$

The above can be deformed to a different appearance as

$$
\frac{d v}{d t}=\frac{r}{I}\left(T-\eta \frac{v^{2}}{r^{2}}\right)
$$

This non-linear pre-rotation system in Eq.(14) can be solved by SIMULINK modelling in MATLAB as shown in Fig.2. Using the data from Boeing 747-400 aircraft, the moment of inertia of real wheel $I=46.19 \mathrm{~kg}-\mathrm{m}^{2}$. The tire radius $r=0.622 \mathrm{~m}$, the landing velocity is $v 0=80.8 \mathrm{~m} / \mathrm{s}$. Pre-rotating torques $T=200 \mathrm{Nm}$ and $500 \mathrm{Nm}$, respectively, are applied. In Fig.3, for a relative low pre-rotating torque, the wheel velocity has not reached the matching speed when the resistance has reached the maximum. In the case of $T=200 \mathrm{Nm}$, the final velocity of wheel can reach to $63 \%$ of the landing speed. i.e. the pre-rotation ratio $\varepsilon=0.63$. The temperature raising energy $\Delta E_{g}$ will be dropped by $86 \%$.
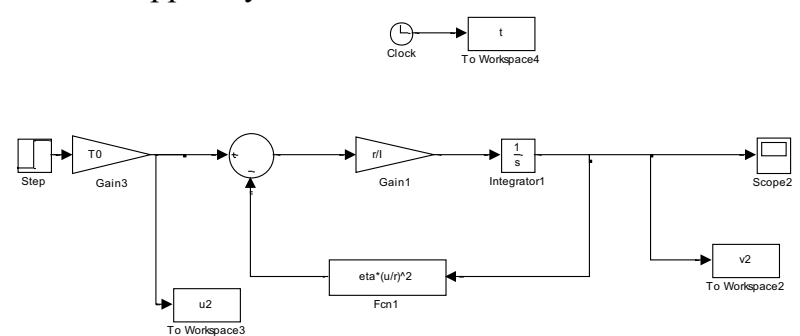

Figure 2. Simulink model for the motion during approaching with non-linear resistance torque

When applying a higher torque $T=500 \mathrm{Nm}$, the wheel will reach the matching speed $100 \%$ within approximately 35 seconds as shown in Fig. 4. Further increasing the torque will be over pre-rotated and is unnecessary.
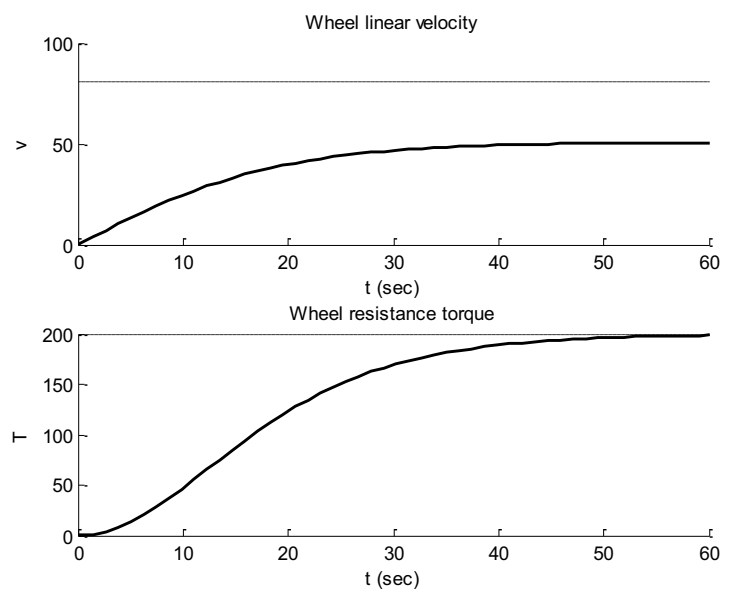

Figure 3. Wheel velocity reaches $63 \%$ of matching speed as resistance torque reaches the peak $(\eta=0.03, T=200 \mathrm{Nm})$

Form Eqs (4-6), when the pre-rotation level is $\varepsilon=1$, it can be seen $t_{s}=0, s_{s}=0$, and $s_{r}=0$ In the full range of $0 \leq \varepsilon \leq 1$, i.e. including all the cases that the pre-rotation exists, does not exist more or less exits, $t_{s}, s_{s}$ and $s_{r}$ have been shown in Fig. 5. Both $t_{s}$ and $s_{s}$ are linear and monotonically declined to zero with the increase of $\varepsilon$. In addition, the friction work at the touch point, wheel kinetic energy increment and total friction work against pre-rotation ratio $\varepsilon$ are shown in Fig. 6. It can be seen, if the pre-rotation is applied by even only $50 \%$, the temperature raising energy caused by the friction work will be reduced by $75 \%$.

\section{Conclusion}

The dynamic model for simulating landing sliding process is established to analyse the phenomenon of aircraft landing smoke and successive tyre wear. The sliding time, distance and friction work under different levels of pre-rotation are presented. Numerical examples with different pre-rotation torques are given. Ideally, a 
$100 \%$ pre-rotation ratio will cancel totally the landing smoke. A partial pre-rotation will have an enlarged effect on the friction heat reduction. The interpretation of the results show that the findings are reasonable and meaningful for understanding the dynamics and energy conversion during the landing, and also for helping the design of the pre-rotation devices. Up to date, the work represents a fundamental approach for understanding the landing smoke problem and an effective solution to it, which is novel and worth further investigation.
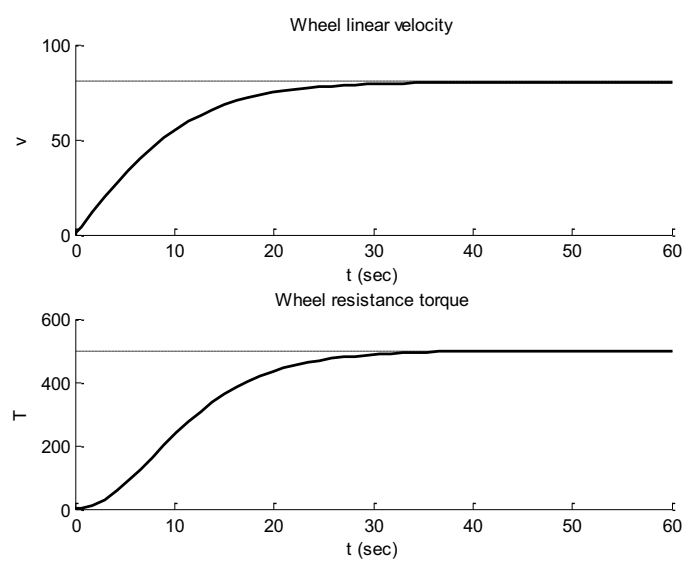

Figure 4. Wheel's tangential velocity has matched the landing speed as resistance torque reaches the maximum $(\eta=0.03$, $T=500 \mathrm{Nm}$ )
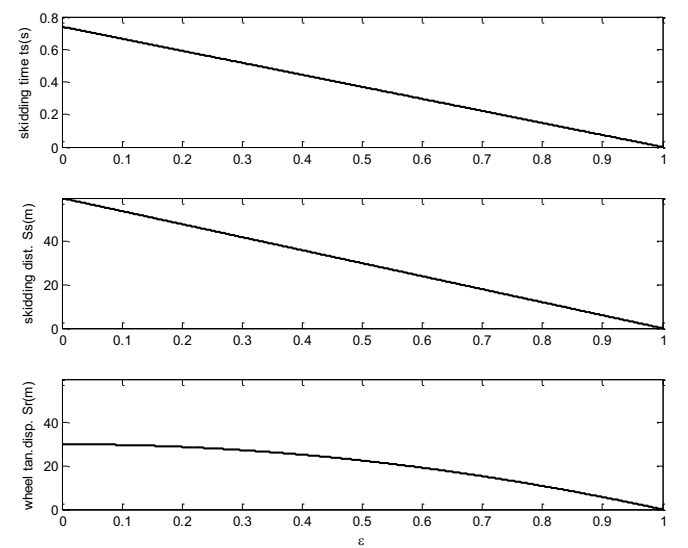

Figure 5. Sliding time, distance and the wheel tangential displacement at touch point.
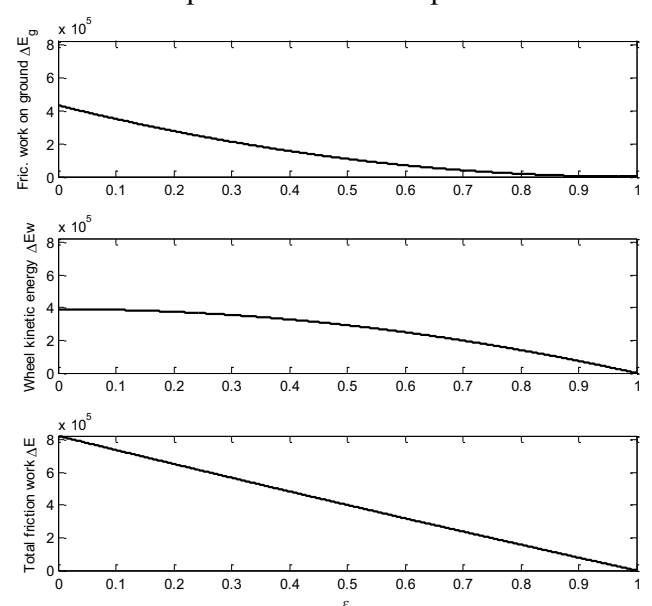

Figure 6. Friction work on the touch point, wheel kinetic energy increment and total friction work

\section{References}

1. Beazley, R. H. Aircraft wheel spinner and control. U.S. Patent, Publication No. US2414849 A. Washington, DC: U.S. Patent and Trademark Office. Retrieved from http://www.google.com/patents/US2414849 (1947).

2. Khal, S., \& Khal, A. Apparatus for pre-rotating aircraft tires. U.S. Patent, Publication No. US20130112809A1, (2013).

3. Boeing Commercial Airplane Co. Approach speeds for Boeing airplanes. Retrieved from http://www.boeing.com/assets/pdf/commercial/airpor ts/faqs/ arcandapproachspeeds.pdf, (2011).

4. Dahl, P. R. A solid friction model. The Aerospace Corporation. Technical report. Los Angeles: Los Angeles Air Force Station. Retrieved from www.dtic.mil/dtic/tr/fulltext/u2/a041920.pdf, (1968).

5. Daidzic, N. E., \& Shrestha, J. Airplane landing performance on contaminated runways in adverse conditions. Journal of Aircraft, 45(6), 2131-2144, (2008).

6. Persson, B. Rubber friction: role of the flash temperature. Journal of Physics: Condensed Matter, 18(32), 7789-7823, (2006).

7. Tong, G., \& Jin, X. Study on the simulation of radial tire wear Characteristics. WSEAS Transaction on Systems, 11(8), 419-429, (2012).

8. Hans B. Pacejka, Tyre and Vehicle Dynamics, Butterworth-Heinemann Ltd, ISBN-10: 0750669187.

9. Rajesh Rajamani, Vehicle Dynamics and Control, Springer.

10. Zuzana Koreňová, Mohammad Juma, et al., Experimental Study of waste Rubber Pyrolysis and Combustion, Petroleum \& Cole, 47(2), 1-19, (2005).

11. Abdurrhman A. Alroqi and Weiji Wang, Comparison of Aircraft Tire Wear with Initial Wheel Rotational Spee, International Journal of Aviation, Aeronautics, and Aerospace, 2(1), (2015).

12. Abdurrhman A. Alroqi and Weiji Wang, Torque of Rotating Device Prior an Airplane Landing, International Journal of Engineering Research and General Science, 2(2), Feb-Mar (2014). 